\title{
An Overview of Activities on Wireless Networks in the European Project COST 257
}

Wojciech Burakowski (Poland), Udo Krieger (Germany), Kenij Leibnitz (Germany), Andrzej Beben (Poland), Michela Meo (Italy), Tolga Ors (United Kingdom), Jorge Garcia-Vidal (Spain), Markus Fiedler (Sweden)

COST 257 Project participants

E-mail:wojtek@tele.pw.edu.pl, leibnitz@informatik.uni-wuerzburg.de

Key words: wireless networks, modelling, performance, network planning, analysis

Abstract: $\quad$ The paper summarises the work on wireless networks inside the COST 257 project, entitled "Impact of new services on the architecture and performance of broadband networks" and chaired by Prof. Tran-Gia of the Technical University of Würzburg (Germany). This project was established for 4 years (1996-2000) and collected the researchers from 16 European countries represented by 32 organisations. Its topics are modelling, performance, network planning and analysis of present and future wireless systems.

\section{INTRODUCTION}

Extending network-based services to wireless mobile subscriber access was an important milestone in the global telecommunication network evolution. Spectacular success of the cellular telephone networks (e.g. GSM - Global System for Mobile) has proven that all, today's and future, network technologies should offer wireless and mobile capabilities.

Enhancement of services supported by the wireless networks is planned to follow evolutionary scenario comprising four main phases, 1 . However, introduction of new services is conditioned by available bandwidth of radio access channel. Today's digital cellular mobile network belongs to the $2^{\text {nd }}$ generation $(2 \mathrm{G})$ of wireless networks offering very low to low bit rate com- 
munication services for voice and data. Further network evolution is on the way towards including packet data services (G2+), Narrow Band Integrated Services Data Network (N-ISDN) capabilities (G3), until, from today's perspective, Broadband ISDN (B-ISDN) services are offered (G4).

A general wireless network architecture assumes three main levels, which are the access network, the core transport network and the service network 2. In the access network, the base station (BTS) provides radio connectivity to the mobile stations (MS). For this purpose, a specialized radio access protocol is implemented in both the base and mobile station. Recognized solutions for such protocols are based on TDMA or CDMA schemes. In the TDMA, for a single MS station a number of time slots in the frame are assigned for the transmission while in the CDMA, all MS stations could generate traffic at the same time using different orthogonal codes 3. Optionally, the access network can contain concentrators (C) to connect a number of BTS stations to the core network. One can distinguish between two types of access networks, indoor and outdoor. In the indoor network the distance between MS and BTS stations is relatively small and typically limited up to 100 meters. On the contrary, the outdoor network covers rather large area, up to few kilometers. The size of this area strongly depends on such parameters as number of MS stations and signal propagation conditions.

The core network is a wide area network that is designated for transporting user and signaling information, and is typically built on cable links (although some wireless links can also be applied, like satellite or radio relay). This network could support a variety of communication services, depending of the involved technology. In general, the access and the core networks are not necessary to be build using the same telecommunication technology. However, for supporting end-to-end network services with adequate quality of service one technology is strongly desirable. For instance, the complete set of broadband services require ATM in both the access and the core network.

The service network contains highly specialized servers that support mobility management and provides application specific processing, e.g. for speech transcoding, voice mail, message and facsimile handling.

In the wireless networks the QoS provisioning problem is more challenging than in the fixed network. This is due to the worst wireless channel characteristics, mainly caused by limited bandwidth and relatively high (and temporary variable) bit error rate. In order to cope with the transmission errors some protection mechanisms are required at the physical or data link layer. However, the implementation of such mechanisms produces additional overheads decreasing effective radio link capacity. As a consequence, availability of resources the network dedicates for a given connection could be variable. Additionally, in the access network a number of connections share the same radio link, and this requires application of special MAC protocols. The problem is 
becoming more complicated when the network additionally supports user mobility. When a customer requires handover, the network should also change the resource allocation to new BTS station.

\section{NARROWBAND NETWORKS}

Present digital cellular systems are considered to be part of the second generation of wireless networks (G2). While these systems were primarily designed to offer voice services, they provide only limited capabilities for data transmission at low and medium bit rates. Most prominent examples for narrowband networks are the Global System for Mobile communications (GSM) in Europe as well as IS-54, IS-95, and Personal Digital Cellular (PDC) in North America and Japan. Additionally, some of the second generation systems like Digital European Cordless Telecommunications (DECT) and Personal Handyphone Systems (PHS) have been introduced to offer wireless services in residential and office environments.

All second generation wireless systems support circuit switched voice and data services with basic rates typically ranging around $9.6 \mathrm{kbps}$. With the growing needs for higher rates, new services are currently being developed that permit multiple allocation to the physical resource. Extensions to GSM like the High Speed Circuit Switched Data (HSCSD) or GPRS (General Packet Radio Service) are considered to be in a transition phase (G2+) towards third generation systems (G3) allowing bit rates up to $170 \mathrm{kbps}$. In addition, enhancements to GSM itself like the introduction of a new modulation scheme under the name of EDGE (Enhanced Data Rates for GSM Evolution) further indicate the evolution of narrowband systems.

\subsection{Planning of Narrowband Wireless Networks.}

Due to today's tremendous customer demand and rapid growths of mobile networks, the need for a systematic planning methodology has become essential. Furthermore, knowledge about customer behavior and measured traffic data allow for detailed planning procedures. In conventional cellular planning, the planning process is driven by radio coverage considerations, i.e., selection of cell site locations, frequency planning, antenna design, etc.



Figure 1 Network planning phases

Basically, the algorithm used for cellular planning consists of four phases, cf. Fig. 1. 
1 In the Radio Network Definition phase, an experienced radio planning engineer chooses cell site locations based on his knowledge and planning experience.

2 Then, the Propagation Analysis evaluates the radio coverage of the area using field strength prediction methods.

3 If the coverage requirements are met, the expected number of traffic channels is calculated and a Frequency Allocation is performed.

4 If the frequency plan can be computed, the network performance is evaluated in the Radio Network Analysis phase by computing some quality of service (QoS) measure in the cells.

From the above description it is apparent that the consideration of the customer traffic influences the network planning approach only to a very limited extent. Thus, a new approach to performing a truly demand oriented network planning has been introduced that takes as input a characterization of customer demand and incoming traffic by discrete points, the demand nodes.

The core component of the new integrated design concept are the automatic network design algorithm Set Cover Base Station Algorithm (SCBPA) and a traffic characterization procedure which generates a set of demand nodes. The first step in this approach is to create a distribution of demand nodes based on estimations of the demand in a certain coverage area. Then, the SCBPA uses a greedy heuristic which selects the optimal set of base stations that maximizes the proportion of covered traffic, i.e., the number of demand nodes which measure a field strength level above a certain threshold value.

One of the key components of the system model for network performance analysis is a model of the demand node pattern. The other major issue is to characterize the behavior of the users at call level and obtain a measure of the performance of the system in terms of subjective Quality of Service (QoS) of the user. Both items will be described in greater detail in the following sections.

\subsection{Description of the Spatial User Demand.}

In this section, we will describe some methods on characterizing the spatial user distribution. At first, we will describe in more detail the notion of a demand node and give a definition as well as a method for generating a demand node pattern from geographical and demographical input data. When such data is unavailable, it is possible to use the realization of a stochastic spatial process. This theoretical approach will also be covered later in this section.

The Demand Node Concept.

A demand node represents the center of an area that contains a quantum of demand from teletraffic viewpoint, 
accounted in a fixed number of call requests per unit, see 4,5 . Such demand nodes represent the user demand discretized both in space and demand.



Figure 2 Geographical and demographical data.



Figure 3 Traffic matrix.



Figure 4 Demand node distribution.

Empirical Distributions.

The sequence of generating a discrete traffic estimation is shown in Fig. 2, 3, and 4. Fig. 2 depicts the input of the estimation algorithm. It contains land usage data that is usually available for the specific area. The colors represent different categories of land usage, e.g. urban, suburban, forest, water, or open areas. Each class is assumed to generate a certain amount of traffic and the traffic matrix is obtained by superimposing the traffic from the different land usage classes active on one area element (Fig. 3). The darker an element of the traffic matrix is, the higher the anticipated demand in the corresponding area element. Then, the matrix representation is being transformed into a spatial discrete point pattern by using a clustering algorithm. A partitioning method has been described in 5. The result of this partitioning is shown in Fig. 4.

Spatial Poisson Process. While the empirical demand node patterns are the most desirable for network planning, often the data is not available to create the traffic matrices. In this case, theoretical stochastic processes are used to describe the user distribution. Such a process is in general a random variable, which takes random choices of mappings from a Borel set to a counting measure, the number of simple points.

The simplest distribution of demand nodes is described by a spatial Poisson point process. In this case the number of points in any Borel set on the plane follows a Poisson distribution, depending on the area of the set and the intensity of the process. If the intensity measure of the process is independent of the location, it is called homogeneous. Such a process is depicted in Fig. 5. 




Figure 5 Spatial Poisson process.



Figure $6 \mathrm{IPhP}^{3}$ process with hyperexponential distribution.

IPhP $^{3}$ process.

The Isotropic Phase Planar Point Process 6,7 allows for characterization of a large number of spatial distributions and can be considered a general case of spatial process. Due to its construction, this process can introduce cluster effects and dependence between sets of points. A realization of the process with hyper-exponential distribution of the inter-point distance is given in Fig. 6. Here, clearly a cluster effect in circular shape can be observed.

2.3. Modeling of Subjective QoS. In this section, we present a basic model aiming at answering the question: How does the cluster structure influence the subjective QoS of a customer in a mobile network? If the network planning does not take into account any customer clustering, the subjective QoS in terms of call blocking probability experienced by a specific test customer will decrease in areas with customer concentration.

For this reason, the cell is described by a finite source model in which the number of sources is obtained from the spatial locations of the customers. In the simple case 4 , if the customer is blocked, he will remain idle. This model is extended in 8 to include the repeated attempt behavior of the customer. The reaction of the user when experiencing call blocking can influence his QoS significantly.

Customer Traffic Process. Fig. 7 shows the model of the cell where the customers can either be in idle or in active states. After call termination or rejection, the user will remain idle until generating the next call. The modeI is therefore a standard loss system with $K$ servers (number of channels) and $X$ sources (random number of customers). The novelty of this approach is to derive the distribution of $X$ from the spatial traffic description. In 4, it is 




Figure 7 Single cell with finite number of customers

shown that there is a degradation of the QoS when the customer population is clustered and the planning did not take such structure into account.

User Retrial Phenomenon. The model can be extended to include a phenomenon quite common in daily life: A user whose call is blocked will immediately retry calling instead of giving up. This causes the network load and thus the blocking probability to rise even further - a snowballing effect of call arrival processes can occur, which leads to dramatic degradations of the call completion performance of single switching systems, and subsequently of the whole network. Fig. 8 shows the modifications of the model from Fig. 7, where $\Theta$ denotes the retrial probability. The analysis is carried out by solving two-dimensional Markov chains in a recursive way (8).



Figure 8 Single cell with finite number of customers and modeling of the retrial phenomenon.

Fig. 9 illustrates the impact of the retrial probability on the blocking probability of first attempts for different ratios of mean call holding time and mean wait-for-reattempt time $\alpha_{0} / \mu$. It can be clearly seen that the blocking probability of the first attempt is higher than without repeated attempt. Comparing to 
the case without retrials $(\Theta=0)$, it is clear that we cannot neglect the repeated attempt phenomenon.



Figure 9 Single cell with finite number of customers.

This phenomenon also holds in a model in which hand-over calls are prioritized over fresh calls by the use of guard channels.

Integrated Services Call Level Model. A service request from a mobile user to a base station may be due either to the generation of a new call or to a handover request. Since handover failures force the termination of a call in progress, these events are considered to be worse than new calls blocking, whose effect is just to force the user to repeat his access request at a later time.

Two mechanisms are implemented in order to favor handover requests over new call requests under heavy traffic conditions ?. First, priority is given to handover requests by reserving a small number of channels for them; second, when the handover of a multimedia call is requested, the base station tries to accommodate the entire call, but, if this is not possible, the two components of the call can be decoupled: the voice connection is accepted, if possible, while the data connection is temporarily suspended, waiting to be resumed as soon as enough channels are available in the cell to serve the entire multimedia call. Decoupling a multimedia call because of a partially served handover can improve the quality of service, mainly because it allows the user to continue at least partly his communication. To facilitate recombination of decoupled multimedia calls, new calls are not accepted as long as some active decoupled connections exist in the cell. 
The cell state, in any instant, is determined by the number of currently active connections for each class of traffic. A continuous-time Markov chain is derived where transition rates are determined from the rates of the arrival processes of new call and handover requests, and from those of the processes of call completion and outgoing handover requests.

As a basic scenario for validation, a configuration with two classes of service was used, comprising voice calls and data calls which require the allocation of 4 channels. Curves showing the average carried traffic for the two classes of service, the average number of busy channels and the average blocking probability for new call and handover requests, were produced using both the analytical model and a simulator. The comparison of the curves clearly indicates that the approximations introduced in the model development do not alter the numerical results significantly.

Other results were also obtained with different configurations of the cell under investigation. Scenarios with voice, data and multimedia connections were considered, with different values of the number of channels required for each data call; the performance of a system in which some channels are reserved to data connections was evaluated, assessing the performance improvements for systems in which the decoupling of multimedia connections is introduced.

\subsection{Dimensioning of Voice Traffic Links.}

Another important aspect in the planning of cellular networks is the appropriate dimensioning of the links transporting the voice traffic. This is especially important when migrating towards future mobile network generations where also data, video, and multimedia services will be supported. In such systems the CDMA access method with variable bit rate vocoders will be employed, resulting in the problem of efficiently transporting streams of low and variable bit rate over a high speed transport network, usually operating with ATM technology.



Figure 10 Packetization of voice packets with AAL2 
The application of ATM Adaptation Layer Type 2 (AAL2) is intended, supporting the multiplexing of several narrowband connections on one ATM connection, see Fig. 10. After encapsulating the voice packet in a CPS PDU, several such CPS packets are fitted into a single ATM cell. In case that the remaining space in the ATM cell is not sufficient to include a CPS packet, its payload is split up in two cells. Additionally, a timer serves to keep the packetization time within a certain delay requirement.

In 9 and 10 studies were performed that investigated the suitability of AAL2 for narrowband CDMA speech connections. Here, the packetization and traffic shaping was modeled as discrete time Markov chain. The transitions were given as recursive state transition equations. From the analysis the distribution of the delay of the speech packet was derived. The results for an E1 link showed that AAL2 is capable of transporting about $80 \%$ of the traffic compared to the traffic without the overhead of AAL2. The reason for this loss in capacity is attributed to the additional CPS packet headers. This example illustrates the need for detalied modeling of the transport network in order to achive a correct dimensioning of the system.

\section{BROADBAND WATM NETWORKS}

In 1996, the ATM Forum Wireless Working Group started activities to develop standards for the wireless ATM network (WATM). Recall that ATM was originally designed for fibre-optic transmission links characterised by extremely low error rate $\left(10^{-12}\right.$ or less). Therefore, adaptation of ATM into the wireless requires updating existing standards by adding radio access layer as well as user/terminal mobility management. These new standards should allow the WATM network to provide the same set of communication services as in the wired network (with similar QOS requirements).

The reference configuration for the WATM network with all WATM application scenarios 11, 12, shows Fig. 11. These configurations differ in terms of terminal mobility, wired or wireless access, mobile or fixed switches, internal ability for establishing ad-hoc networks and co-operation level with PCS (Personal Communication Systems) systems.

The configurations \# 1 and \# 2 refer to two distinct components of WATM: the radio access technology, and enhancement of existing ATM technology to support end-system mobility, respectively. The rest of recommended configurations correspond to the enhancement WATM for supporting mobile switches, building ad-hoc networks, supporting PCS access and internetworking PCSATM.

The WATM network demands an enhanced protocol stack comparing to cable ATM network. The required protocol stack, which should be implemented in the terminals, access points and/or ATM switches, depends on the assumed 




Figure 11 Reference configuration for WATM

network configuration. These enhancements are related with wireless access and mobility management. Wireless access provisioning requires some modifications on the physical layer while supporting mobility demands new functions implemented at the ATM layer and in the signaling protocol.

The reference protocol stack to cope with wireless and mobility is depicted on Fig. 1212.



Figure 12 Reference protocol stack

The objective of the RAL (Radio Access Layer) layer is to support data transport over radio links with satisfactory quality. It includes the wireless 
MAC, LLC and PHY layers. The RAL may be generic, designed to support multiple higher protocols such as ATM, IP, etc.

Between regular ATM and RAL layers the ATM CL (Convergence Layer) layer is added. The objective of this sublayer is to provide ATM cell conversion to the format acceptable for RAL layer.

Supporting mobility management demands some enhancements in signaling protocols. Additionally, in the mobile terminal local M-ATM layer should be implemented for supporting different mobility aspects, e.g. handover, terminal tracking, security etc.

3.1. Data link layer issues. Considering the transport of messages in an advanced packet-switched network at the data link, network interface and network layers over an error-prone communication channel, e.g. a wireless ATM network, the transport characteristics are additionally influenced by handover procedures according to the implemented terminal mobility management 13.

Furthermore, the hierarchical structure of the existing protocol stack, the segmentation of messages and the correlation of errors at the wireless physical layer as well as the error recovery at the wireless LLC layer have a strong impact on the transport performance.

The network interface layer, called block layer here, coincides with the ATM adaptation layer and provides a service-specific bearer service over an errorprone communication channel for the network and transport layers on top of it, for example LLC/SNAP encapsulated IP frames (cf. 14, Sec. 4.1.2, p 202f). An Automatic-repeat-request (ARQ) protocol such as Selective Repeat (SR) or Go-back-N (GBN) and their ramifications can be applied both at the wireless data link layer as well as at the service-specific convergence sublayer (SSCS) or at the common part convergence sublayer (CPCS) of the ATM adaptation layer (AAL) to cope with the defective transport of ATM cells and to guarantee a secure delivery of messages to the higher layers cf. 15, 16, 17.

3.1.1 Objectives. Regarding the efficiency of the data transport a large block length $n$ is required while error considerations demand short blocks. Therefore, we suppose that the size of the block PDUs can be varied to some extent, that the transport channel of cell PDUs is error prone due to noise, failures of the transmission equipment, data loss, as well as customer and terminal mobility and that a Go-back-N (GBN) ARQ protocol with window size $m$ is applied at the block layer to guarantee a correct delivery of the block PDUs free from losses, cf. 17, p. $129 f$.

The question arises how the performance of a GBN protocol subject to the segmentation of messages and the correlated errors of the PDUs, particularly the additional load due to retransmissions, can be determined by ana- 
lytic means and how the corresponding parameters should be selected. Then it may be possible to perform a self-tuning of the block length and window size processes under the control of the error detection process at the cell level.

3.1.2 Analysis of the Go-Back-N performance. To analyze the impact of correlated errors at the block and cell levels on the performance of the GBN ARQ protocol running at the block level we assume in accordance with observations in real networks, that the transport errors of consecutive blocks $b_{i}, b_{i+1}, i \geq 1$, are correlated (cf. 15, 16, 18, 19). We assume that a cell at position $j, 1 \leq j \leq n$, in $b_{i+1}$ is damaged with probability $s$ if its counterpart was already damaged at position $j$ in $b_{i}$. With probability $p$ it is transported without an error if the cell at the same position in the previous block was received without an error (see Fig. 13).



Figure 13 Correlation of errors during the cell transport

The corresponding error process at the cell level is modeled by a binary discrete-time Markov chain (DTMC) $\left\{X_{j}(t), t \geq 0\right\}$ on a probability space $(\Omega, \mathcal{K}, \mathbb{P})$ with state space $\Sigma=\{0,1\}$ and the states $X_{j}(t)(\omega)=0, \omega \in \Omega$, for error-free and $X_{j}(t)(\omega)=1$ for defective cell transport of the $t$-th block. $X_{j}(0)$ represents some initial condition of the process. The DTMC is described by a transition probability matrix (t.p.m.)

$$
P_{C}=\left(\begin{array}{cc}
p & 1-p \\
1-s & s
\end{array}\right)=\left(\begin{array}{ll}
p & q \\
r & s
\end{array}\right)
$$

with $q=1-p, r=1-s, 0<p<1,0<s<1$ (see Fig. 14).



Figure 14 Markov model of a single cell error process 
However, a more complicated correlation structure depending on the block length $n$ by some function $\chi(n) \in \mathbb{N}$ can be incorporated into a similarly structured two-state model using the t.p.m.

$$
P_{C}(n)=\left(\begin{array}{ll}
p(n) & q(n) \\
r(n) & s(n)
\end{array}\right)=\left(\begin{array}{cc}
\hat{p} & \hat{q} \\
\hat{r} & \hat{s}
\end{array}\right)^{\chi(n)}
$$

with $\hat{q}=1-\hat{p}, \hat{r}=1-\hat{s}, 0<\hat{p}<1,0<\hat{s}<1$, e.g. $\chi(n)=n+1$ yields

$$
\begin{aligned}
P_{C}(n)= & \left(\begin{array}{ll}
\hat{p} & \hat{q} \\
\hat{r} & \hat{s}
\end{array}\right)^{n+1} \\
= & \frac{1}{\hat{r}+\hat{q}}\left[\left(\begin{array}{ll}
\hat{r} & \hat{q} \\
\hat{r} & \hat{q}
\end{array}\right)\right. \\
& \left.\quad+(1-\hat{r}-\hat{q})^{n+1}\left(\begin{array}{cc}
\hat{q} & -\hat{q} \\
-\hat{r} & \hat{r}
\end{array}\right)\right]
\end{aligned}
$$

and reflects a nonlinear dependence of the correlation structure on the block length (cf. 15).

In the following we illustrate the proposed approach using only the simplest model. To simplify the analysis and to proceed to a tractable model, the individual error processes $X_{j}, 1 \leq j \leq n$, at each cell position $j$ in consecutive blocks are assumed to be independent of each other and in steady state. Applying an aggregation-disaggregation argument, its behavior is approximated by a t.p.m. of the form

$$
\Gamma(n)=\left(\begin{array}{ll}
p^{n} & 1-p^{n} \\
\omega(n, r, p) & 1-\omega(n, r, p)
\end{array}\right) \in \mathbb{R}^{2 \times 2}
$$

with

$$
\omega(n, r, p)=\frac{1}{2^{n}-1} \cdot\left[(r+p)^{n}-p^{n}\right]>0
$$

where the range of $s, p \in(0,1)$ has to be limited such that $0<\omega(n, 1-$ $s, p)<1$. The steady-state block error probability of the aggregated DTMC $Z(t)$ is given by:

$$
\begin{aligned}
\epsilon & =\epsilon(n, r, p)=\mathbb{P}\{Z=1\} \\
& =\frac{1-p^{n}}{1-p^{n}+\omega(n, r, p)} \in(0,1)
\end{aligned}
$$

We assume that the return channel sending the positive and negative acknowledgements of the ARQ protocol is not disturbed, that the transport channel for block PDUs is working in a slotted manner where a PDU transmission 
time constitutes one time slot, and that for a window size $m$ the round-trip propagation delay is constant and equal to an integral number $m-1$ of time slots, cf. 15. Then the mean delay

$$
D=\frac{m}{\eta}
$$

of the block transport (cf. 15, (2.10), p. 232, 16, (30), p. 725). and the throughput efficiency $\eta$ of the Go-Back-N protocol with window size $m$ for the aggregated correlated error process $\{Z(t), t \geq 0\}$ in steady state with the t.p.m. $\Gamma(n)$ are determined by:

$$
\eta(n, s, p)=\left[1+\frac{m\left(\omega(n, 1-s, p)+1-p^{n}\right) \cdot\left(1-p^{n}\right)}{\omega(n, 1-s, p)\left[1-\left(p^{n}-\omega(n, 1-s, p)\right)^{m}\right]}\right]^{-1}
$$

$\eta(n, s, p)$ is a function of the window size $m$, the block length in terms of the number $n$ of consecutive cells and the transition probabilities $p$ between errorfree cell transports and that one $s$ between defective transports, respectively.

Some results are shown in Fig. 15 and 16. Here we assume that the probability $\left(P_{C}\right)_{10}=1-s=r$ of a transition from an unsuccessful to a successful cell transport between consecutive blocks is given by $r=s=0.5$ and the probability $\left(P_{C}\right)_{01}=1-p=q$ of a transition from a successful to an unsuccessful cell transport between consecutive blocks as well as the number $n$ of cells per block are varied.



Figure 15 Throughput efficiency of GBN ARQ for varying transition probability $q$ and window size $m=4$

The sketched model can be used as first simple approach to study the impact of varying block length, segmentation and correlated errors on the performance of an ARQ protocol.

3.2. Satellite MAC protocols. In this section we analyze the performance of an Adaptive Random-Reservation Medium Access 




Figure 16 Block delay of GBN ARQ for varying transition probability $q, r=0.5$, message length $n \in\{2,3,7,15\}$ and window size $m=4$

Control (MAC) protocol which can support all ATM service classes while providing the required Quality of Service (QoS).

Our study focuses on parameter optimisation of the multiple access schemes for ATM over a GEO satellite with on-board processing capabilities, considering various traffic mixes of CBR (Constant Bit Rate), $\mathrm{rt}-\mathrm{VBR}$ (real time Variable Bit rate), nrt-VBR (non-real time Variable Bit Rate) and UBR(Unspecified Bit Rate).

It is shown that maximum throughput can be achieved by using this access scheme. A TDMA access protocol combining both Random Access and Demand Assignment Multiple Access (DAMA) is particularly suited for a scenario with a high number of terminals with very bursty UBR traffic (e.g. web browsing). UBR sources with short burst length access the slots remaining after the reservation procedure by random access which drastically reduces the slot access delay, at the expense of lower utilisation. However for UBR sources with burst sizes consisting of several ATM cells, reservation access provides higher throughput but the access delay is considerable longer.

The adaptive MAC protocol was designed to allow statistical multiplexing of ATM traffic over the air interface, especially for the independent and spatially distributed terminals. It is shown that the potential user population which can be served is considerably increased by statistically multiplexing bursty traffic over the air interface.

\subsubsection{Framework for proposed MAC protocol.}

Design objectives. The MAC protocol has to be designed to allow statistical multiplexing of ATM traffic over the air interface, especially in the uplink for the independent and spatially distributed terminals. The following design objectives are taken into consideration: 
- maximize the slot utilization, especially for bursty traffic

- guarantee the QoS requirements for all service classes

- maximize frame efficiency by minimizing overheads

The minimization of overheads is not an easy task, especially for ATM which was designed for channels with very good error characteristics (Bit Error Rates around $10^{-10}$ ). To minimise cell loss over the satellite link, channel coding has to be used to make the transmission more robust. A LLC header to facilitate error recovery mechanisms is optional and not in scope of this study. Finally a satellite specific header with satellite routing and wireless resource management fields is added to form a MAC packet as shown in Fig. 17.



Figure 17 Encapsulation of ATM Cells to MAC Packets and mapping to TDMA Frame

Access schemes. MAC layer access schemes can be typically categorised into four classes: Fixed Access, Random Access, Demand Assignment Multiple Access (DAMA) and Adaptive Access. The first three techniques have evolved to meet the needs of constant high traffic with long duration's, sporadic traffic with short to medium duration's, and sporadic traffic with long duration's, respectively. Finally adaptive access is used to meet the needs of multiple media which consists of traffic with various characteristics. Thus to meet the design objectives an Adaptive Access mechanism seems to be the best choice.

Mapping of ATM service classes onto MAC service classes. To simplify the conceptual design of the MAC protocol, ATM service classes 20 can be mapped onto MAC service classes.

Mapping of CBR service category. Fixed-Rate DAMA is ideal for connections with a constant bit rate such as the CBR service class in ATM 
networks. Before a connection is set-up, the terminal and satellite negotiate the Quality of Service (QoS) parameters. These QoS parameters determine the characteristics of the connection. Since the parameters will not be modified during the connection, the amount of bandwidth allocated for that connection will not be changed until the connection is terminated. For ATM CBR connections the Peak Cell Rate is allocated to the terminal.

Mapping of rt-VBR service category. Real-time Variable Bit Rate services can also be supported with fixed-rate DAMA. For real-time services, the amount of bandwidth assigned to the connection should be close or equal to the Peak Cell Rate (PCR) to avoid cell delay. The major drawback of this scheme is that a major portion of the bandwidth is wasted when the cell transfer rate is lower than the assigned bandwidth. The major difficulty to employ variable-rate DAMA in ATM satellite systems is the effect of the large propagation delay. The computing and negotiation process between the satellite and the terminal may be too long for rt-VBR services and result in unacceptable QoS. The use of variable-rate DAMA for rt-VBR is only possible if the arriving traffic can be predicted one hop delay in advance. Since this is not possible except in some special cases fixed-rate DAMA will be used for rt-VBR. A scenario where fixed-rate DAMA is efficient for rt-VBR services is when the terminal can multiplex traffic from multiple services. In this case the aggregate traffic can be approximated as a constant cell flow by using a small amount of shaping.

Mapping of nrt-VBR service category. VBR services which are not time sensitive can be assigned an effective bandwidth which is between the mean cell rate and PCR. Since the required bandwidth of VBR sources changes with time, there may be instants when the cell transfer rate is higher than the amount of bandwidth (effective bandwidth) assigned to that connection. In this case cells can be buffered in the terminal and in case the queue exceeds a certain threshold more bandwidth can be requested. Thus using variable-rate DAMA the bandwidth of a connection can be adjusted according to the change of the data transfer rate.

Mapping of UBR service category. No numerical commitments are made for the UBR service class and this service category is intended for nonreal time applications. UBR services could be supported by variable-rate DAMA. However the fact that this service class has the lowest priority (because no commitments to CLR are made) has to be considered. We propose that UBR could transmit data directly to the unoccupied data slots without reservation. The unreserved slots are broadcasted on the downlink to be accessed by random 
access. This is particularly appealing for bursty interactive services with short duration, for which the long slot reservation delay is unacceptable.

\subsubsection{The random-reservation adaptative assignment pro-} tocol. The TDMA frame of the adaptive assignment protocol is divided into Reservation slots, Control slots, Data slots and Random Access slots, as shown in Fig. 18. The protocol is based on the proposals by 21, 22, 23 with modifications to achieve the design objectives for multi-service networks.



Figure 18 Frame Structure

A Reservation slot is that period of time in which terminals report their requests to the reservation unit. There are only a few Reservation slots available and a terminal selects one at random without knowing whether another station is using the same slot. If more than one terminal selects the same reservation slot, a collision occurs and terminals have to retransmit after waiting for a mean retransmit waiting time determined by the collision resolution algorithm. The MAC protocol ensures that the collision probability stays low. The reason for using reservation slots is because ATM networks support different services which have different loss and delay requirements.

If a single request was received for a reservation slot (successful request), the on-board wireless resource management module tries to allocate the necessary Data slots. If no Data slots are available, the request can either be blocked (called blocking probability) or queued. We propose to queue successful requests in a prioritised queue so that the terminal does not need to compete with other terminals for a reservation slot again. By queuing successful reservations, requests can be allocated data slots according to their priorities.

Once the Data slots are reserved (successful reservation), an acknowledgement is transmitted to the terminal in TDM mode on the downlink frame. Data slots represent the part of the frame in which a terminal can transmit its message after a successful reservation. In every frame there are many data slots and the on-board wireless resource management module will assign data slots to a 
particular successful request. A data slot is assigned to at most one terminal and therefore there is no possibility of collision.

On the other hand Random Access (RA) slots represent the part of the frame in which terminals can transmit without the need of making a reservation. The slots available for random access are broadcasted in the downlink frame. This part is for services which don't want to wait for the lengthy reservation procedure. In random access mode it is not possible to guarantee a certain QoS to users although the protocol will try to minimise the number of collisions to maximise throughput by using an adaptive collision resolution algorithm. Random Access should only be used by UBR sources with relatively small burst length since RA terminals are not allowed to reserve slots and have to content for each MAC packet.

Unless the number of reservation slots per frame is carefully adjusted the result would be either low capacity utilisation and long delays (too many reservation slots, less capacity available for information transmission) or network backlog (too few reservation slots resulting in successive collisions and high delay). The number of reservation slots should be fixed for system behaviour where the number of collisions can be controlled by broadcasting a message in the downlink that services with lower priority should not send/resend requests till the collisions have been resolved. Our analysis has shown that two reservation slots provide adequate performance. However when the number of collisions can't be controlled new reservation slots can be added by reducing the number of control slots.

The requests for dynamic slot allocation are done using the Control Slots which are assigned, on a round-robin basis to all terminals which request the variable-rate DAMA MAC class. The number of control slots is set to eight to minimise the frame overhead.

The satellite frame introduces a constant delay equal to the frame length, on the cells of a stream connection. Therefore the selection of the frame size should be small enough to satisfy the delay limit of real-time services $(400 \mathrm{~ms})$ 24 taking into account the satellite propagation and processing delays and the delay introduced by the terrestrial B-ISDN.

The MAC packet slot period has been chosen to support a $32 \mathrm{kbit} / \mathrm{s}$ CBR stream and corresponds to one frame unit of 384 un-coded information bits every uplink frame. This results in a frame period of $11.9 \mathrm{~ms}$ to transmit 84 ATM cells per second using AAL5. There are 64 MAC packet slots to support 2.048 $\mathrm{Mbit} / \mathrm{s}$ of traffic per spot-beam on the uplink. The actual uplink transmission rate is higher due to ATM and MAC layer overheads.

3.3. Cell level. The ATM network services are precisely defined in 20 and they are: CBR, VBR, ABR and UBR. WATM network should offer these services with the required QoS parameter values as shown in Table 
111. These parameters correspond to the ATM cell level QoS parameters and they were defined from the point of view of the user application.

Table 1 QoS parameters for specific ATM services

\begin{tabular}{|c|c|c|c|}
\hline & CBR, rt-VBR & nrt-VBR & ABR \\
\hline CLR & $10^{-7}-10^{-4}$ & $10^{-9}-10^{-6}$ & $10^{-9}-10^{-4}$ \\
\hline CDV & $<1 \mathrm{~ms}$ & not specified & not specified \\
\hline max CTD & $2 \mathrm{~s}-10 \mathrm{~ms}$ & $10 \mathrm{~s}-500 \mathrm{~ms}$ & not specified \\
\hline PCR & $10 \mathrm{Mbps}-32 \mathrm{kbps}$ & & $10 \mathrm{Mbps}-9.6 \mathrm{kbps}$ \\
\hline SCR & $6 \mathrm{Mbps}-32 \mathrm{kbps}$ & $6 \mathrm{Mbps}-32 \mathrm{kbps}$ & \\
\hline BT & $5 \mathrm{Mbps}-2 \mathrm{kbps}$ & $1 \mathrm{Mbps}-1 \mathrm{kbps}$ & \\
\hline
\end{tabular}

3.3.1 Evaluation of CBR, VBR and UBR services. In this section we summarise the results presented in 25,26 concerning the performances of the ATM network services in the fixed wireless environment (reference configuration \#1). The assumed configuration is a bottleneck topology. The tested connections of different type were established between two terminals, each of them connected to the network by a RAP (Radio Access Point) unit. Furthermore is assumed that the radio access to the network is governed by the MEDIAN protocol 27 and transmission errors occur independently.

Transmission errors. The radio channels are usually characterised by relatively high BER value, even up to $10^{-2} 28$. The situation is going worst when the terminal is on the move and then the transmission conditions can be temporary worst due to fading and shadowing $28,29,30$. As a consequence, even that a powerful protection mechanism is used the cells could be lost even in bursts.

MAC protocol. A number of new MAC protocols for the purpose of the wireless ATM network was recently submitted, among them the most recognised are MASCARA 28, SAMBA 31 and MEDIAN 27. These protocols were designed to operate under TDMA (Time Division Multiple Access) scheme, where the time slot allocation for a given connection is made dynamically. In the MEDIAN, the time slots are assigned to the active connections on the basis of the polling information and the connection priority. The priority strictly corresponds to the maximum permitted cell waiting time $\left(\Delta_{\max }\right)$. A cell is discarded when it waits longer than $\Delta_{\max }$. Notice that a cell from higher priority connections could be served after serving the cell of lower priority. Therefore, this protocol does not support the possibility to reserve a fixed number of slots in consecutive transmission frames, what is extremely required e.g. for CBR connection. 
Exemplary numerical results (partially verified by measurements) were obtained by using OPNET package environment. Based on them one can conclude as follows:

CBR service. Additional mechanisms should be implemented in order to support CBR service. One solution is to adjust the $\Delta_{\max }$ parameter in the MEDIAN protocol. By setting larger value of the $\Delta_{\max }$ one can expect larger $\mathrm{CDV}$, but the cell loses can be avoided. On the contrary, low value of the $\Delta_{\max }$ leads to low CDV, but cell loss ratio increases. Another possible solution is to keep large $\Delta_{\max }$ value and to provide a buffer at the connection end point. This buffer introduces fixed delay but the cells are delivered to the destination with constant rate and very low CDV. In $32,33,34$ some propositions for a playback buffer management scheme for the CBR service in the fixed ATM network were submitted. As it was expected these solutions fail in the case of WATM network due to high bit error rate and large CDV. In 26 we propose appropriate modifications for the playback buffer mechanism. The proposed enhancements were related to the strategy of inserting dummy cells instead of lost ones and extension $\mathrm{K}$ times cell numbering scheme by assigning the same number to $\mathrm{K}$ consecutive cells.

VBR service. Satisfying the requirements from Table 1 it seems to be difficult to reach. The features of the MEDIAN protocol, which introduces too large delay, mainly cause this. However, from the application viewpoint, these relatively large CTD values (about $23 \mathrm{~ms}$ ) are still acceptable (see Table 235). Anyway, a similar mechanism, like it was proposed for the CBR service, to reduce $\mathrm{CDV}$, is extremely desirable.

Table 2 Allowed values of $\max$ CTD for various types of applications

\begin{tabular}{|l|c|c|}
\hline \multicolumn{1}{|c|}{ Application } & Bandwidth & Max CTD \\
\hline Voice & $8-16 \mathrm{kbps}$ & $150 \mathrm{~ms}$ \\
\hline Videotelephony & $64 \mathrm{kbps}-2 \mathrm{Mbps}$ & $350 \mathrm{~ms}$ \\
\hline Videoconference & $128 \mathrm{kbps}-14 \mathrm{Mbps}$ & $350 \mathrm{~ms}$ \\
\hline Transaction processing & $64 \mathrm{kbps}-5 \mathrm{Mbps}$ & $3 \mathrm{~s}$ \\
\hline Telescript session & $4-5.6 \mathrm{Mbps}$ & $250 \mathrm{~ms}$ \\
\hline
\end{tabular}

TCP over UBR Service. The TCP greedy source was used to evaluate the effectiveness of UBR service. The gathered cell transfer characteristics correspond to throughput, window size and RTT. In the running experiments the bit error rate was $7.5 * 10^{-3}$ (for higher bit error rate the TCP segment lost probability was too high). The cell loss ratio (CLR) for this BER was 
$10^{-2}$. The obtained results say than even assuming so heavy conditions, the effectiveness of cell transfer is still acceptable.

\subsubsection{ABR flow-control. Connections of the ABR service class} are designed to use the remaining capacity of an ATM network after the CBR and VBR service-class connections have received their current cell rate. To avoid congestion in an ATM network and to use the whole capacity efficiently, the source of every ABR connection has to be informed regularly about this remaining capacity. For this purpose, the ATM Forum has standardized ratebased ABR flow-control algorithms.

Considering the high-speed data transfer by the ABR service class in a wireless ATM (WATM) network, the impact of forward and backward hard handover protocols on the performance of the two rate-based ABR flow-control schemes Explicit Rate Indication for Congestion Avoidance improvement (ERICA + ) and Fuzzy Explicit Rate Marking Adaptation (FERMA) has been investigated (cf. 36, 37, 38. For this purpose, the ABR service class that is specified in the ATM forum document Traffic Management Specification 4.0 is used (cf. 20). The underlying WATM architecture and the used protocol stack of this study are derived from NEC's prototype implementation WATMnet and the ATM forum documents (cf. 39, 40, 41). It includes a simplified version of the TDMA/TDD structure and the associated wireless MAC protocol of this system. Using a model of a basic client-server scenario in a wireless ATM network, the impact of the error-prone wireless communication channel and of mobility-management techniques determined by backward and forward hard handover protocols has been studied (cf. 39, 42, 43, 44, 45).

3.4. Handover protocols. Regarding data communication in a WATM network the connections must be permanently maintained during the communication phase since a mobile terminal (MT) moves within a certain coverage area of a base station (BS) and may cross its boundary (cf. $39,42,43,44,45)$. In this case, the connections must be handed over to a new transmission cell whereby a new radio channel is seized and the QoS requirements of the corresponding virtual channels (VCs) must be satisfied in addition to the existing ones within the new cell. The corresponding hard handover protocol has to guarantee the sequence integrity and loss-free delivery of the ATM cells during this transition process. Moreover, interworking with the rate-based $\mathrm{ABR}$ flow-control algorithms is required to guarantee the effectiveness of the approach (see 36, 38 and references therein, also section 3.3.2).

To achieve these goals, an improved backward hard handover protocol has been developed for handovers between the transmission cells of the current BS (CBS) and a new BS (NBS) of the MT, called better hard handover (see Fig. 
19 for a simplified error- and loss-free message transfer without acknowledgements - cf. 36).



Figure 19 Better Hard Handover

Derived from a congestion-awareness concept the new handover protocol is a minimal enhancement of a standard hard handover protocol. It includes only a few new signaling messages using special RM cells. Independent of handover events they are exchanged between the base stations and the mobile switching center (MSC) which is an ATM switch with mobility support enhancements for the wireless part of the network. Whenever there is a substantial change of the ABR bandwidth or an alteration in the number of active $\mathrm{ABR}$ connections in the transmission area of a BS the BS informs the MSC immediately by sending an MSC information message with the current $\mathrm{ABR}$ target cell rate TCR, the number of ABR connections and the sum of their minimum cell rates. This very low additional bandwidth requirement in the path between each BS and the MSC gives the MSC the knowledge about the current possible explicit rate (ER) in each BS transmission area.

The improved backward hard handover is combined with forward handover as a fallback procedure in the case of a too short announcement period before a handover. This means that normally every mobile terminal announces its expected new handover $t_{a}$ milliseconds before the real handover event occurs. If there is not enough time for such an announcement, the mobile terminal has to perform a forward handover (see Fig. 20, cf. 36).

\section{WIRELESS LANS}

In ad-hoc WLANs, where every station has a similar functionality, Carrier Sense Multiple Access (CSMA) based protocols may be advantageous because of their simplicity and because no station is compelled to assume special functions. Recently proposed standards such as the IEEE 802.11 or HIPERLAN I use variations of the basic CSMA scheme. 


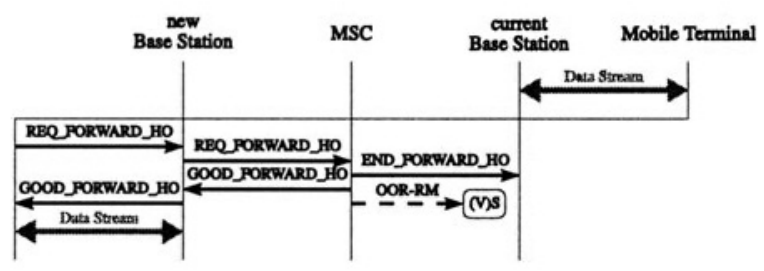

Figure 20 Forward Handover

However, if we are to integrate delay-sensitive and delay-insensitive traffic in the same WLAN, we must keep end-to-end delay and delay variations below certain bounds for the delay-sensitive traffic. Unfortunately, it is well known that in general CSMA protocols suffer from large access delay quantiles (the basic CSMA algorithm is actually unstable) due to the existence of packet collisions.

This section describes FIFO by Sets CSMA (FS-CSMA) 46, a MAC protocol for high-speed ad-hoc WLANs. FS-CSMA is a Collision Resolution Algorithm that builds groups (Transmission Sets) with the packets arrived during fixed-length time intervals. Packets belonging to the same Transmission Set are transmitted using CSMA with random backoff whereas the different Transmission Sets are served following a FIFO discipline.

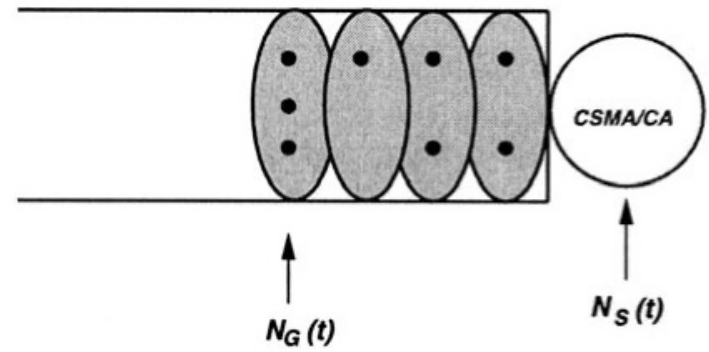

Figure 21 FS-CSMA as a distributed FIFO queueing system

FS-CSMA generates a collection of (possibly empty) sets called Transmission Sets (TSs) $\left\{S_{k}\right\}$, each of them containing the packets arrived during successive disjoint grouping intervals of constant length $W$ slots. These Transmission Sets are served (i.e. their included packets are transmitted using CSMA) with a FIFO discipline, so that $S_{k}$ is served before $S_{j}$ if $k<j$. If every $S_{k}$ had no more than one packet to be transmitted we would be facing a FIFO-MAC algorithm, with packets being sent in order of arrival.

The number of packets per TS is closely related to the integer $W$, which determines the length of the grouping interval, and to the network load $\rho$. The smaller $W$ or $\rho$ are the more the system behaves like a FIFO system. On the 
contrary, the protocol will perform as pure CSMA if $W$ is made large enough. The value of $W$ must be chosen as a trade-off between the desired properties of a FIFO scheduling and the overhead introduced by the algorithm.

An FS-CSMA ad-hoc network may be seen as a distributed FIFO queueing system (see Fig. 21). The elements of this distributed queue are the Transmission Sets and their service consists of the successful transmission via CSMA with random backoff of all the packets included in the Transmission Set.

\section{REFERENCES}

[1] H. Nakamura. H. Tsuboya and A. Nakajima. Applying ATM to Mobile Infrastructure Networks. IEEE Communication Magazine. 36(1): 67-73.1998

[2] N. Gerlich. The Impact of Wireless Communication on Broadband Network Architecture and Performance. Proceedings of the COST 257 Mid-Term Seminar. Jan.1999.

[3] T. Ojanpera and R. Prasad. Wideband CDMA for third generation mobile communications.

Artech House Publishers. 1998.

[4] P. Tran-Gia and N. Gerlich. Impact of Customer Clustering on Mobile Network Performance. COST 257 document. 1997.[257TD(97)07]

[5] T. Leskien K. Tutschku. and P. Tran-Gia. Traffic estimation and characterization for the design of mobile communication networks. COST 257 document. 1997. [257TD(97)22].

[6] M.-A. Remiche. Efficiency of an iphp ${ }^{3}$ illustrated through a model in cellular networks. COST 257 document. 1998. [257TD(98)13].

[7] M.-A. Remiche. Impact of non-stationary users distribution on the outage probability measure in cdma wireless system. [257TD(99)01].

[8] Tran-Gia and M. Mandjes. Modeling of customer retrial phenomenon in cellular mobile networks.IEEE Journal on Selected Areas of Communications, 15(08):1406-1414, Oct. 1997. [257TD(97)06].

[9] K. Elsayed N. Gerlich, P. Tran-Gia. and N. Jain. Performance analysis of a link carrying capacity in cdma systems. COST 257 document. 1997. [257TD(97)22].

[10] N. Gerlich and M. Menth. The performance of aal-2 carrying cdma voice traffic. COST 257 document. 1998. [257TD(98)32].

[11] L. Dellaverson. Wireless atm requirements specification. ATM Forum. Draft Specification. 01.03. Feb.1999, ATM Forum, Feb. 1999.

[12] B. Rajagopalan. Draft wireless atm capability set 1 specification. Draft Specification. 01.12, ATM Forum, Sep. 1999. 
[13] G. Carle and E. Dorner. Assessment of edge devices with error control mechanisms for ATM networks with wireless local loop. Personal Wireless Communications. Aachener Beitrage zur Informatik. Verlag Augustinus Buchhandlung. 1996.

[14] D. Ginsberg. ATM solutions for enterprise interworking. Addison-Wesley, New York, 1996.

[15] C. H. C. Leung and et al. The throughput efficiency of a go-back-n arq scheme under Markov and related error structures. IEEE Trans. on Comm., 36(2):231-234, 1988.

[16] D. L. Lu and J. F. Chang. Performance of arq protocols in nonindependent channel errors. IEEE Trans. on Comm., 41(4):721-730, 1993.

[17] T. N. Saadawi and et al. Fundamentals of Telecommunication Networks. John Wiley \& Sons. 1994. New York.

[18] H. Ohta and T. Kitami. A cell recovery method using fec in atm networks. IEEE Journal on Selected Areas in Communications. 9(9):1471-1482, 1991.

[19] G.R. Pieris and G.H. Sasaki. Performance of the go-back- $\infty$ protocol under correlated packet losses. IEEE Trans. on Comm., 41(5):660-663, 1993.

[20] N. Giroux. Traffic management specification version 4.0. Specification. 4.0, ATMForum April. 1996.

[21] A.K. Elhakeem S. Bohm. and V.K.M. Murthy. Analysis of a movable boundary random/dama accessing technique for future integrated services satellites. IEEE Globecom, pages 1283-1289, 1993.

[22] N. Celandroni and E. Ferro. The foda-tdma satellite access scheme: Presentation, study of the system and results. IEEE Trans. Comms. 39(12): 1823-1831, 1991.

[23] M. Tondriaux T. Zein, G. Maral. and D. Seret. A dynamic allocation protocol for a satellite network integrated with b-isdn. Proc. of 2nd ECSC, pages 15-20, 1991.

[24] ITU-T. B-ISDN ATM layer cell transfer performance. Recommendation, I.356 ITU-T. 1996

[25] W. Burakowski A. Bak, A. Beben and Z. Kopertowski. On quality of watm network services. COST 257 document, 1999. [257TD(99)11].

[26] Beben and W. Burakowski. On improving cbr service playback buffer mechanisms in watm network. COST 257 document. 1999. [257TD(99)12].

[27] MEDIAN Partners. Median final system design. Interim project deliverable, 1996.

[28] G. Marmigcre L. Merakos F. Bauchot, S. Decrauzat. and N. Passas. Mascara, a mac protocol for wireless. Interim project deliverable.

[29] B.Sklar. Rayleigh fading channels in mobile digital communication systems. part I: Characterization, IEEE Communication Magazine, September 1997.

[30] B.Sklar. Rayleigh fading channels in mobile digital communication systems. part II: Mitigation. IEEE Communication Magazine, September 1997.

[31] ACTS 2004 Program SAMBA. Samba - System for advanced mobile broadband application. Interim project deliverable.

[32] B. Bharucha M. Noorchashm. and G. Wetzel. Buffer design for constant bit rate services in presence of cell delay variation. ATM Forum document. af95-1454, ATM Forum, 1995. 
[33] J. Virtamo (Eds.) J. Roberts. U. Mocci. Broadband network teletraffic. Performance evaluation and design of broadband multiservice networks, Final report of action COST $242,1996$.

[34] ITU-T. B-Isdn atm adaptation layer (aal) specification. Recommendation. I.363.

[35] M. Niemi. Application requirements for watm. ATM Forum document. af96-1058, ATM Forum, 1996.

[36] U.R. Krieger and M. Savoric. Performance evaluation of handover protocols for data communication in a wireless atm network. Proc of ITC, 1999.

[37] U. R. Krieger and M. Savoric. The Performance of abr flow-control algorithms and flow control protocols in a wireless atm network. COST 257 document. 1998.[257TD(98)31].

[38] Udo R. Krieger and M. Savoric. Performance evaluation of handover protocols for data communication in a wireless atm network. COST 257 document, 1998. [257TD54].

[39] K.Rauhala. Baseline text for wireless atm specifications. Draft Specification. btd-watm01.07, ATM Forum, April 1998.

[40] J. Porter and et al. The orl radio atm system, architecture and implementation. Intermin report, Olivetti research Ltd, January 1996.

[41] D.C. Cox. Wireless personal communications: What is it? IEEE Personal Communications, pages 20-35, April. 1995.

[42] K. Y. Eng E. Ayanoglu. and M. J. Karol. Wireless atm: Limits, challenges and proposals. IEEE Personal Communications, pages 18-34. August1996..

[43] G.P. Pollini. Trends in handover design. IEEE Communications Magazine, pages 82-89, March. 1996.

[44] R. Ramjee and et al. Performance evaluation of connection rerouting schemes for atmbased wireless networks. IEEE/ACM Transactions on Networking, 6(3), 1998.

[45] C.K. Toh. Wireless ATM and Ad-Hoc Networks. Kluwer Academic Publishers, Boston 1997.

[46] Vázquez-Cortizo. D. and Garcia. A collision resolution algorithm for ad-hoc wireless lan, Broadband Communications, pages 119-130, 1998.

[47] Ch. Bulloch. Ka-band Abroad: The world rises to the challenge, Via Satellite, March 1997.

[48] R. Fernandez. The Ka-band quest continues, Via Satellite, March 1997.

[49] E.F. Fitzpatrick. Hughes Spaceway: Wireless interactive broadband service, Proc. of Second Ka-Band Utilization Conference, September 24-26, 1996.

[50] T. Ors. S. Sun and B.G Evans. A meshed vsat satellite network architecture using an onboard atm switch, IEEE IPCCC, 208-215, February 1997.

[51] W.R. Stevens. TCP/IP illustrated, Addison-Wesley. 1995.

[52] B. Jabbari. G. Colombo. A. Nakajima and J. Kulkarni. Network issues for wireless communications, IEEE Communications Magazine, pages 88-98, January 1995.

[53] D. Raychaudhuri and et al. WATMnet: A prototype wireless atm system for multimedia personal communication, IEEE Journal on Selected Areas in Communications, 15(1): 83-95, 1997 
[54] R. Jain and et al. ERICA Switch Algorithm: A complete description, ATM Forum document, af96-1172, ATM Forum August 1996.

[55] U. R. Krieger and M. Savoric. Adaptation of the fuzzy explicit rate marking to abr flowcontrol in a wireless atm network, Proc. of MMB, September 1999. 